\title{
Constipation-Predominant Irritable Bowel Syndrome Associated to Hyperprolactinemia
}

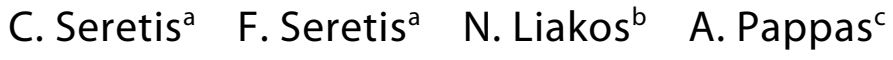 \\ D. Keramidaris ${ }^{b} \quad$ S. Gourgiotis ${ }^{b} \quad$ N. Salemis ${ }^{b}$ \\ E. Lagoudianakis ${ }^{b}$
}

aGastrointestinal Endoscopy Department and ${ }^{\mathrm{b}} 2$ nd Department of Surgery, 401 General Army Hospital of Athens, Athens, and 'Internal Medicine Department, Argos Hospital, Argos, Greece

\section{Key Words}

Prolactin · Cholecystokinin · Irritable bowel syndrome · Constipation

\begin{abstract}
Irritable bowel syndrome (IBS) is considered to be a physical disorder that mainly affects the bowel and is clinically characterized by lower abdominal pain or discomfort, diarrhea, constipation (or alternating diarrhea/constipation), gas, bloating, and nausea. According to recent studies, it appears that there is an association with increased prolactin levels in patients suffering from IBS. We report a rare case of regression of IBS symptoms (constipation type) in a 16-year-old female adolescent after receiving cabergoline for treating hyperprolactinemia due to pituitary macroadenoma. Our hypothesis is that increased prolactin levels, for instance due to a pituitary adenoma, may suppress prolactin-releasing peptide release and lead to a reverse feedback interaction, consequently resulting in oversecretion of cholecystokinin, inducing the development of IBS.
\end{abstract}

\section{Introduction}

Irritable bowel syndrome (IBS) is the most common of all gastrointestinal disorders, affecting around $15 \%$ of the population at least in Western societies. The division of IBS into subgroups is based on the fact that these patients behave in dissimilar ways. IBS subgroups building on stool and defecation patterns can be divided into diarrheapredominant (D-IBS), constipation-predominant (C-IBS) and alternating (A-IBS) subtypes [1]. From recent studies, it appears that cholecystokinin (CCK) levels are statistically significantly higher in patients with IBS [2]. CCK belongs to the group of 
endogenous molecules known as 'brain-gut neuropeptides'. CCK is found in the nerves of the circular muscle layer of the colon and to some extent in the ileum, and it is abundant in the celiac plexus and vagus nerves [3]. Due to these multiple anatomical locations it is not surprising that CCK has been implicated in different physiological functions: gallbladder contraction and pancreatic enzyme secretion, as well as motor and sensory functions at various levels of the gastrointestinal tract such as gastric emptying and colonic motility [4]. Further studies have shown that CCK also acts as a mediator of the gastrocolonic response [5, 6], while CCK infusion appears to unmask intestinal dysmotility in patients with IBS [7]. Except for this, new studies suggest that there is a strong correlation between CCK and the prolactin-releasing peptide (PrRP), since peripheral administration of CCK is proven to activate neurons expressing PrRP [8]. As a result, increased prolactin levels, for instance due to a pituitary adenoma, may suppress the secretion of PrRP and lead to a reverse feedback, consequently resulting in oversecretion of CCK, inducing the development of IBS. Nevertheless, to the best of our knowledge, there are no studies regarding the CCK levels in pathological hyperprolactinemia that estimate the possible benefit of regression of IBS after receiving dopaminergic agonists for treating hyperprolactinemia. We report a unique case of confronting C-IBS symptoms in a female adolescent who was under cabergoline as a treatment of pituitary macroprolactinoma.

\section{Case Report}

A 16-year-old female addressed the Emergency Department complaining of acute abdominal pain of the periumbilical area without reporting any other symptoms. History examination revealed the presence of similar episodes in the past few months, most of the times relieved with defecation, which remained undiagnosed despite full-scale examinations, including colonoscopy and abdominal CT. The patient also mentioned that, since the last year, she suffered of constipation, reporting 1-2 movements per week. In addition, she reported catamenial disorders (complete disarrangement of the catamenial circle) since the previous year, without dysmenorrhea. The following complete physical examination and laboratory tests did not reveal the existence of an underlying cause concerning the abdominal pain. Abdominal X-ray revealed mediocre dilatation of the intestinal loops, without other pathological findings. The patient underwent abdominal and gynecological ultrasound examination without any pathological findings. In the meantime the pain spontaneously regressed completely. Due to the complete lack of pathological signs, and taking into consideration the reported catamenial disorders, a full endocrinological test set was performed, revealing prolactin levels of $456.04 \mathrm{ng} / \mathrm{ml}$. In consequence, a pituitary gland MRI was performed, revealing a pituitary macroadenoma $(1.2 \times 1 \times 1 \mathrm{~cm})$, confirming the endocrinological results (fig. 1). The patient then was submitted to treatment with cabergoline. Prolactin levels returned to normal after almost 3 years since the beginning of the treatment, a fact that was mainly attributed to the patient's reluctancy to follow the treatment plan closely. The initial cabergoline dose was set at $1 \mathrm{mg} /$ week and was gradually elevated to $4 \mathrm{mg} /$ week. The response to the dose was evaluated by regular measurement of prolactin levels every 6 months. During the period the patient was receiving cabergoline, she noticed a dramatic decrease of the cramping abdominal pain episodes, from 10-12 before the administration of cabergoline to only 3 after the submission to the particular treatment. Moreover, she mentioned that her bowel habits had been absolutely normalized, with almost daily defecation. The length of this period seems to be reasonable to suggest regression of the IBS symptoms, which can be attributed to cabergoline, through the pathophysiological pathways presented.

\section{Discussion}

Prolactin has many effects, including regulating lactation through stimulation of the mammary glands to produce milk (lactation), and provides the body with sexual 
gratification after sexual acts. Prolactin also stimulates the proliferation of oligodendrocyte precursor cells. It also has a number of other effects, including contributing to surfactant synthesis of the fetal lungs at the end of pregnancy and to immune tolerance of the fetus by the maternal organism during pregnancy; it also decreases the normal levels of sex hormones - estrogen in women and testosterone in men. In addition, it appears that prolactin has a crucial role as an immunoregulator affecting the development of the neonatal intraepithelial $\mathrm{T}$ lymphocytes found between the epithelial cells of the small intestine [9]. Furthermore, the expression of prolactin transcripts has also been clearly observed in intraepithelial lymphocytes purified from mouse intestine [10]. From this point of view, it can be explained that prolactin levels significantly increase in active celiac disease [11] as well as in colorectal cancer [12], with research suggesting that prolactin may be a reliable biomarker regarding the activity and severity of these two conditions. We must take into account that there are indications giving reason to believe patients with IBS have 'low-grade intestinal inflammation', and the increase in $\mathrm{T}$ lymphocytes and mast cells has been associated with disorders found in IBS, such as the communication between the intestine and the nervous system, the increase in intestinal permeability and changes in the microbiota [13]. Therefore, it can be suggested that prolactin levels can reasonably rise in these patients.

Regarding the correlation of CCK and PrRP, CCK is released from the gut in response to meals and has an important regulatory role in gastrointestinal function, inhibiting gastric motility and emptying via a neural reflex within the caudal brainstem or via a reflex loop that ascends to the hypothalamus via a relay in the caudal brainstem [14, 15]. Peripheral CCK acts on afferent nerve fibers of the gastric vagus nerve; these vagal neurons project to the caudal brainstem [16], where they activate neurons that project to hypothalamic nuclei involved in appetite regulation [14, 15]. In particular, peripheral injections of CCK activate neurons in the nucleus tractus solitarii and ventrolateral medulla, including specific subpopulations of the noradrenergic neurons of the A1 and A2 cell groups [17]. These noradrenergic neurons comprise several subpopulations, which are anatomically, biochemically and functionally separate. Some of these noradrenergic neurons express PrRP [18]. For this reason, prolactin can be directly associated to the regulation of gastrointestinal mobility. Our hypothesis is that increased prolactin levels, for instance due to a pituitary adenoma, may suppress PrRP release and lead to a reverse feedback interaction, consequently resulting in oversecretion of CCK, inducing the development of IBS. In order to reach safe conclusions, further studies are needed to directly describe the interaction between hyperprolactinemia and CCK, especially after administrating dopaminergic agonists, so as to technically decrease prolactin levels, and estimate the possibility of their use as a treatment option in IBS.

\section{Conclusions}

Increased prolactin levels are proven to be significantly higher in patients suffering from C-IBS. An interesting case of regression of IBS symptoms in a patient with prolactinoma, after receiving cabergoline for treating hyperprolactinemia, is presented. The close relation of prolactin and CCK seems to have a crucial role regarding the regulation of intestinal mobility and could possibly indicate future therapeutic perspectives. 


\begin{tabular}{r|l|l|l}
$\begin{array}{r}\text { Case Reports in } \\
\text { Gastroenterology }\end{array}$ & $\begin{array}{l}\text { Case Rep Gastroenterol 2011;5:523-527 } \\
\text { Dol: 10.1159/000331806 }\end{array}$ & $\begin{array}{l}\text { Published online: } \\
\text { September 3, 2011 }\end{array}$ & $\begin{array}{l}\text { O 2011 S. Karger AG, Basel } \\
\text { ISSN 1662-0631 } \\
\text { www.karger.com/crg }\end{array}$ \\
\hline
\end{tabular}

\section{Disclosure Statement}

The authors declare that they have no competing interests.

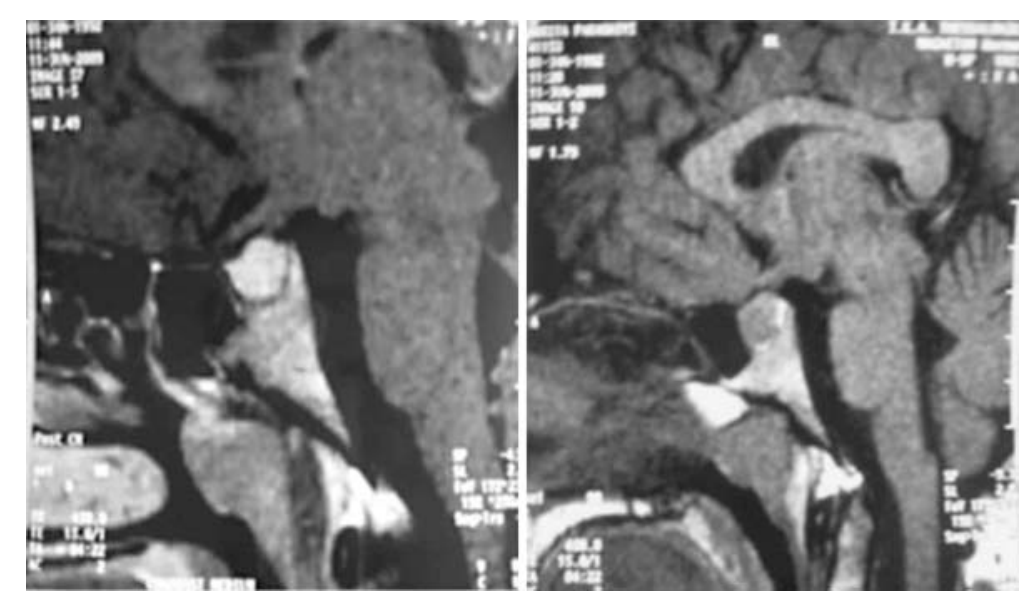

Fig. 1. MRI image of the pituitary adenoma: the adenoma measured $1.2 \times 1 \times 1 \mathrm{~cm}$, therefore it was classified as a macroadenoma. There were no signs of suppression of the optic chiasm, in accordance to the lack of relative neurological symptoms.

\section{References}

1 Eriksson E, Andrén K, Eriksson H, Kurlberg G: Irritable bowel syndrome subtypes differ in body awareness, psychological symptoms and biochemical stress markers. World J Gastroenterol 2008;14:4889-4896.

2 Zhang H, Yan Y, Shi R, Lin Z, Wang M, Lin L: Correlation of gut hormones with irritable bowel syndrome. Digestion 2008;78:72-76.

3 Liddle RA: Cholecystokinin; in Walsh JH, Dockray GJ (eds): Gut Peptides. Biochemistry and Physiology. New York, Raven, 1994, p 175.

4 Crawley JN, Corwin RL: Biological actions of cholecystokinin. Peptides 1994;15:731-755.

5 Renny A, Snape WJ Jr, Sun EA, London R, Cohen S: Role of cholecystokinin in the gastrocolonic response to a fat meal. Gastroenterology 1983;85:17-21.

6 Harvey RF, Read AE: Effect of cholecystokinin on colonic motility and symptoms in patients with the irritablebowel syndrome. Lancet 1973;1:1-3.

7 Kellow JE, Phillips SF, Miller LJ, Zinsmeister AR: Dysmotility of the small intestine in irritable bowel syndrome. Gut 1988;29:1236-1243.

8 Lawrence CB, Ellacott KL, Luckman SM: PRL-releasing peptide reduces food intake and may mediate satiety signaling. Endocrinology 2002;143:360-367.

9 Urtishak S, McKenna E, Mastro M: Prolactin and prolactin receptor expression in rat, small intestine, intraepithelial lymphocytes during neonatal development. Dev Immunol 2001;8:319-330.

10 Nagano M, Chastre E, Choquet A, Bara J, Gespach C, Kelly PA: Expression of prolactin and growth hormone receptor genes and their isoforms in the gastrointestinal tract. Am J Physiol 1995;268(3 Pt 1):G431-G442.

11 Kapur G, Patwari AK, Narayan S, Anand VK: Serum prolactin in celiac disease. J Trop Pediatr 2004;50:37-40.

12 Soroush AR, Zadeh HM, Moemeni M, Shakiba B, Elmi S: Plasma prolactin in patients with colorectal cancer. BMC Cancer 2004;4:97. 
13 Ortiz-Lucas M, Saz-Peiró P, Sebastián-Domingo JJ: Irritable bowel syndrome immune hypothesis. Part one: the role of lymphocytes and mast cells. Rev Esp Enferm Dig 2010;102:637-647.

14 Schwartz GJ: Integrative capacity of the caudal brainstem in the control of food intake. Philos Trans R Soc Lond B Biol Sci 2006;361:1275-1280.

$\checkmark 15$ Broberger C, Hökfelt T: Hypothalamic and vagal neuropeptide circuitries regulating food intake. Physiol Behav 2001;74:669-682.

16 Maruyama M, Matsumoto H, Fujiwara K, et al: Prolactin-releasing peptide as a novel stress mediator in the central nervous system. Endocrinology 2001;142:2032-2038.

17 Luckman SM: Fos-like immunoreactivity in the brainstem of the rat following peripheral administration of cholecystokinin. J Neuroendocrinol 1992;4:149-152.

$\checkmark 18$ Chen C, Dun SL, Dun NJ, Chang JK: Prolactin-releasing peptide-immunoreactivity in A1 and A2 noradrenergic neurons of the rat medulla. Brain Res 1999;822:276-279. 